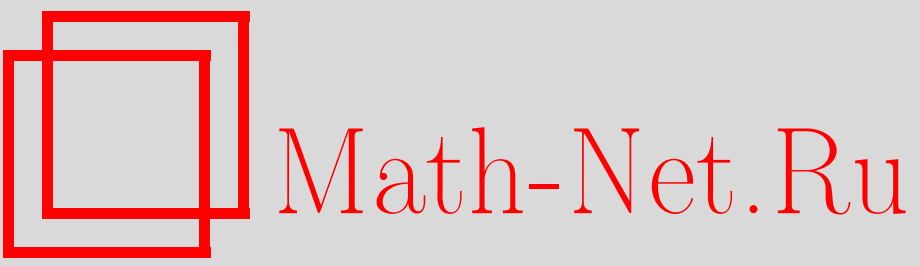

Н. А. Бойкова, Н. Е. Нюнько, Ю. Н. Тюхтяев, Р. Н. Фаустов, Трехмерный релятивистский подход к описанию эффектов отдачи в водородоподобных системах частиц с разными массами, ТМФ, 2002, том 132, номер $3,339-348$

DOI: https://doi.org/10.4213/tmf366

Использование Общероссийского математического портала Math-Net.Ru подразумевает, что вы прочитали и согласны с пользовательским соглашением

http: //www.mathnet.ru/rus/agreement

Параметры загрузки:

IP: 3.85 .5 .30

26 апреля 2023 г., $07: 26: 08$ 
ТЕОРЕТИЧЕСКАЯ

И МАТЕМАТИЧЕСКАЯ

ФИЗИКА

Том 132, № 3

сентябрь, 2002

(C) 2002 г.

Н. А. Бойкова* , Н. Е. Нюнько*, Ю.Н. Тюхтяев ${ }^{*}$, Р.Н. Фаустов ${ }^{\dagger}$

\section{ТРЕХМЕРНЫЙ РЕЛЯТИВИСТСКИЙ ПОДХОД К ОПИСАНИЮ ЭФФЕКТОВ ОТДАЧИ В ВОДОРОДОПОДОБНЫХ СИСТЕМАХ ЧАСТИЦ С РАЗНЫМИ МАССАМИ}

Описан метод получения логариффмических по отношению масс поправок к $S$-уровням энергии водородоподобного атома. С его помощью впервые аналитически рассчитан ряд новых поправок подобного типа.

Ключевые слова: водородоподобные атомы, мюоний, энергетическая структура, сдвиги уровней, квазипотенциал, функция Грина, амплитуда рассеяния, инфракрасные особенности, эффекты отдачи.

Легкие водородоподобные (ВП) атомы - классический объект для исследований,позволяюший подвергать результаты квантовой теории очень точной экспериментальной проверке. Важнейшую роль в развитии релятивистской теории связанных состояний играет уравнение Дирака. Из этого уравнения следует наличие спина и спинового момента у электрона и позитрона и непосредственно вытекают задачи о тонкой и сверхтонкой структуре энергий ВП-атомов. Точное решение уравнения Дирака с кулоновским потенциалом является отправной точкой для определения поправок к сдвигам уровней энергии и к величинам их сверхтонкого расщепления. Уравнение Дирака с радиационными поправками [1], предложенное Швингером, позволяет учитывать многократное взаимодействие частицы с собственным и внешним полями.

Важное место в современном развитии теории связанных состояний занимает метод эффективного уравнения Дирака. В этом методе возможен последовательный переход от двухчастичной теории к приближению внешнего поля [2], [3], однако ввиду отсутствия симметрии между частицами в ВП-атоме возникают сложности при рассмотрении систем, подобных позитронию. Универсальным и симметричным в описании обеих частиц является квазипотенциальный подход [4]. Подробное изложение современного состояния теории связанных двухчастичных систем дается в обзорах [5], [6]. Аналитические выражения для поправок к уровням энергии ВП-атомов являются функциями

\footnotetext{
* Саратовский государственный университет, Саратов, Россия

† Научный совет по комплексной проблеме "Кибернетика" РАН, Москва, Россия
} 
малых параметров - $\alpha$ (константы тонкой структуры) и отношения масс $\beta=m_{1} / m_{2}$ частиц, образующих атом. Наиболее значительными по величине оказываются поправки, содержащие логарифмы констант $\alpha^{-1}$ и $\beta^{-1}$.

В настояшее время факт отсутствия вклада $\left(m_{1}^{2} \alpha^{6} / m_{2} n^{3}\right) \ln \alpha^{-1}$ в тонкий сдвиг $S$-уровней энергии ВП-атома можно считать надежно установленным (здесь и далее $m_{1}$ и $m_{2}$ - массы легкой и тяжелой частиц ВП-атома, соответственно, $n$ - главное квантовое число). Решающий шаг в этом направлении был сделан в работах Фелла, в частности в работе [7], где метод и техника вычислений изложены наиболее детально. Сравнение формул (2)-(4) работы [7] с выражениями для расчета поправок к уровням энергии мюония от процессов обмена одним поперечным фотоном, записанными на основе квазипотенциального метода [8], [9], обнаруживает значительную аналогию подходов. Более того, в работе [10] было показано, что единственным интегралом, приводяшим к логарифмическим вкладам в сверхтонкое расщепление основного уровня энергии ВП-атома, является стандартный интеграл, названный так потому, что техника выгисления других логарифмических по $\alpha$ поправок сводится к сравнению соответствуюших аналитических выражений с этим интегралом,

$$
\begin{aligned}
& I_{\mathrm{c \textrm {T }}}^{1 S}=\frac{1}{8 \pi^{2}} \iint \frac{d \vec{p} d \vec{q}}{\varepsilon_{1 p} \varepsilon_{2 p}} \varphi_{p}^{2}(\omega) \varphi_{q}^{2}(\omega) \frac{p^{2} q^{2}}{\vec{p}_{q}^{2}}=\frac{\pi^{2}}{2 m_{1} m_{2}} \ln \alpha^{-1}, \\
& I_{\mathrm{cT}}^{2 S}=\frac{1}{8 \pi^{2}} \iint \frac{d \vec{p} d \vec{q}}{\varepsilon_{1 p} \varepsilon_{2 p}} \varphi_{p}^{3}(\gamma) \varphi_{q}^{3}(\gamma) \frac{p^{2} q^{2}}{\vec{p}_{q}^{2}}\left(p^{2}-\gamma^{2}\right)\left(q^{2}-\gamma^{2}\right)=\frac{\pi^{2}}{2 m_{1} m_{2}} \ln \alpha^{-1},
\end{aligned}
$$

где

$$
\begin{gathered}
\omega=\alpha \mu, \quad \mu=\frac{m_{1} m_{2}}{m_{1}+m_{2}}, \quad \vec{p}_{q}=\vec{p}-\vec{q}, \\
\varphi_{p}(A)=\left(p^{2}+A^{2}\right)^{-1}, \quad \varepsilon_{i p}=\sqrt{p^{2}+m_{i}^{2}}, \quad \gamma=\frac{\omega}{2} .
\end{gathered}
$$

Конечный интеграл (24) работы [7], вычисленный Феллом при определении пропорциональной $\ln \alpha^{-1}$ поправки, имеет вид

$$
I=\iint \frac{d p d q}{\varepsilon_{1 p} \varepsilon_{2 p}} \varphi_{p}^{3}(\gamma) \varphi_{q}^{3}(\gamma)\left(p^{2}-\gamma^{2}\right)\left(q^{2}-\gamma^{2}\right)\left(p^{2}-q^{2}\right)^{2} \frac{p^{2} q^{2}-(\vec{p} \vec{q})^{2}}{|\vec{p}-\vec{q}|^{6}}
$$

В таблице C.3 приложения D работы [7] он выписан под номером 6. Опуская в подынтегральном выражении (3) величины, не содержащие $\ln \alpha^{-1}$, можно произвести замену

$$
\left(p^{2}-q^{2}\right)^{2}\left[p^{2} q^{2}-(\vec{p} \vec{q})^{2}\right]\left|\vec{p}_{q}\right|^{-6} \rightarrow \frac{1}{2} \frac{p^{2} q^{2}}{\vec{p}_{q}^{2}},
$$

в результате чего интеграл $I$ сводится к интегралу $I_{\text {ст }}^{2 S}(2)$,

$$
\begin{aligned}
m_{1} m_{2} I & =\frac{1}{2} \iint \frac{d \vec{p} d \vec{q}}{\vec{p}_{q}^{2}} \varphi_{p}^{3}(\gamma) \varphi_{q}^{3}(\gamma) p^{2} q^{2}\left(p^{2}-\gamma^{2}\right)\left(q^{2}-\gamma^{2}\right)= \\
& =4 \pi^{2} I_{\text {ст }}^{2 S}\left(\varepsilon_{i p} \rightarrow m_{i}\right)=2 \pi^{4} \ln \alpha^{-1}
\end{aligned}
$$


При $\varepsilon_{i p} \rightarrow m_{i}$ интеграл Фелла (2) расходится в области больших импульсов. Однако именно в таком виде он приведен под номером 3 в таблице C.3 приложения D в работе [7]. Обсуждая в качестве простого примера интеграл

$$
\int_{0}^{\infty} p d p \varphi_{p}(\gamma) \varphi_{p}(m)=\frac{1}{m^{2}-\gamma^{2}} \ln \frac{m}{\gamma}=\frac{1}{m^{2}} \ln \frac{m}{\gamma}\left[1-\frac{\gamma^{2}}{m^{2}}+\cdots\right]
$$

Фелл пришел к выводу, что можно отыскать вклад от этого интеграла, пропорциональньй $\ln \alpha$, рассмотрев промежуток интегрирования $\gamma<p<m$, который он назвал логарифмическим. В самом деле,

$$
\int_{\gamma}^{m} \frac{d p}{p}=\ln \frac{m}{\gamma}
$$

Таким образом, поведение подынтегрального выражения при больших импульсах в случае вычисления вкладов, пропорциональных $\ln \alpha$, оказывается несущественным. Однако корректное определение стандартного интеграла представляет несомненный интерес.

Рассмотрим незначительную трансформацию стандартного интеграла (1),

$$
i_{\mathrm{cT}}=\frac{1}{8 \pi^{2}} \iint \frac{d \vec{p} d \vec{q}}{\varepsilon_{1 p} \varepsilon_{2 p}} \varphi_{p}^{2}(\omega) \varphi_{q}^{2}(\omega) \frac{p^{4}}{\vec{p}_{q}^{2}}
$$

Выделим главный по $\alpha$ вклад

$$
i_{\mathrm{c \textrm {T }}}=\frac{\pi}{2 \omega} \int_{0}^{\infty} \frac{d p}{\varepsilon_{1 p} \varepsilon_{2 p}}=\frac{\pi}{2 \omega m_{2}} \int_{0}^{\infty} \frac{d p}{\sqrt{p^{2}+1} \sqrt{p^{2}+\beta^{2}}} .
$$

Этот интеграл - полный эллиптический интеграл первого рода и не выражается в элементарных функциях. Однако из него легко выделяется основной логарифмический по малому параметру $\beta$ вклад

$$
i_{\mathrm{c \textrm {T }}}=\frac{\pi}{2 \omega m_{2}} \int_{0}^{\infty} \frac{p d p}{\sqrt{p^{2}+1}\left(p^{2}+\beta^{2}\right)} \cong \frac{\pi}{2 \alpha m_{1} m_{2}} \ln \beta^{-1} .
$$

Таким образом, при вычислении вкладов, пропорциональных $\ln \beta^{-1}$, важно сохранять радикалы $\varepsilon_{i p}$, обеспечивающие сходимость интегралов.

В работах [11], [12], положивших начало современному этапу исследования проблемы тонкого сдвига уровней энергий ВП-атомов, был получен результат

$$
\begin{aligned}
\Delta E_{\mathrm{T}}= & \frac{z^{5} \alpha^{5} \mu^{3}}{\pi n^{3} m_{1} m_{2}}\left\{\frac{2}{3} \delta_{l_{0}} \ln \alpha^{-1}-\frac{8}{3} \ln \left[K_{0}(n)\right]-\right. \\
& \left.-\frac{2}{m_{2}^{2}-m_{1}^{2}}\left[m_{2}^{2} \ln \frac{m_{1}}{\mu}-m_{1}^{2} \ln \frac{m_{2}}{\mu}\right]+C\right\}
\end{aligned}
$$

где $\ln \left[K_{0}(n)\right]$ - логарифмм Бете, а $C$ - константа, определяющая величину вклада порядка $\alpha^{5}$. В целом выражение (10) имеет порядок $\alpha^{5}$, однако наряду с малой константой $\alpha$ в него входит другая малая величина $\beta$. Это обусловливает поправку чрезвычайно высокого порядка

$$
\frac{\alpha^{5} \mu^{3}}{m_{1} m_{2}} \beta^{2} \ln \beta^{-1}
$$


Трехмерный релятивистский подход к описанию связанных состояний двух частиц [8]-[10], [13] позволяет обнаружить новые разнообразные логарифмические по параметру $\beta$ поправки к величине тонкого сдвига $S$-уровней энергии ВП-атомов в пятом и шестом порядках по константе тонкой структуры и выяснить условия, при которых вклад, найденный в работах [11], [12], оказывается единственным. Для исследования взаимодействий в ВП-атоме введем квазипотенциал $\widetilde{V}$ с помошью амплитуды $\tilde{\tau}_{c}$,

$$
\widetilde{V}(\vec{p}, \vec{q} ; E)=\tilde{\tau}_{c}-\tilde{\tau}_{c} \widehat{G}_{c}^{+} \tilde{\tau}_{c}+\cdots,
$$

где

$$
\begin{gathered}
\tilde{\tau}_{c}=\left[\widehat{G}_{c}^{+}\right]^{-1} \widehat{G_{c} \widetilde{T} G_{c}^{+}}\left[\widehat{G}_{c}^{+}\right]^{-1}, \\
\widehat{G}(\vec{p}, \vec{q} ; E)=\int \frac{d p_{0} d q_{0}}{(2 \pi)^{2}} G\left(p_{0}, \vec{p}, q_{0}, \vec{q} ; E\right), \\
\widehat{G}^{+}(\vec{p}, \vec{q} ; E)=u_{1}^{*}(\vec{p}) u_{2}^{*}(-\vec{p}) \widehat{G}(\vec{p}, \vec{q}, E) \Gamma_{0} u_{1}(\vec{q}) u_{2}(-\vec{q}), \\
G_{c}=G_{0}\left(1+T_{c} G_{0}\right), \quad \Gamma_{0}=\gamma_{10} \gamma_{20}, \quad T_{c}=K_{c}\left(1+G_{c} K_{c}\right) .
\end{gathered}
$$

Волновая функция двухфермионной системы с полной энергией $E$ удовлетворяет квазипотенциальному уравнению

$$
\left\{\left[\widehat{G}_{c}^{+}(\vec{p}, \vec{q} ; E)\right]^{-1}-\tilde{V}(\vec{p}, \vec{q} ; E)\right\} \psi_{E}(\vec{q})=0 .
$$

Решая это уравнение по теории возмушений, в низшем порядке находим

$$
\varphi_{c}^{\prime}(\vec{p})=\Omega_{p} \varphi_{c}(\vec{p}), \quad \Omega_{p}=\frac{M_{p_{1}} M_{p_{2}}}{2 \mu} E_{p}^{+},
$$

где $E_{p}^{+}=\left(E+\varepsilon_{1 p}+\varepsilon_{2 p}\right)^{-1}, M_{p i}=\varepsilon_{p i}+m_{i}, \varphi_{c}(\vec{p})$ - решение нерелятивистского уравнения Шредингера с кулоновским потенциалом. Необходимость модификации фейнмановской диаграммной техники легко объяснима. Двухвременная кулоновская функция Грина представляется в виде

$$
\widehat{G}_{c}=\widehat{G}_{0}\left(1+K_{c} \widehat{G}_{c}\right)=\left(1+\widehat{G}_{c} K_{c}\right) \widehat{G}_{0},
$$

где $K_{c}=v_{c} \Gamma_{0}, v_{c}-$ кулоновский потенциал,

$$
\begin{gathered}
\widehat{G}_{0}(\vec{p}, \vec{q} ; E)=\left(\Lambda^{++} F-\Lambda^{--} F^{\prime}\right) \Gamma_{0}, \quad \Lambda^{ \pm \pm}(\vec{p})=\Lambda_{1}^{ \pm}(\vec{p}) \Lambda_{2}^{ \pm}(-\vec{p}), \\
\Lambda_{i}^{ \pm}=u_{i}^{ \pm} \otimes u_{i}^{* \pm} u_{i}^{ \pm}, \quad F=(2 \pi)^{3} \delta(\vec{p}-\vec{q})\left(E-\varepsilon_{1 p}-\varepsilon_{2 p}\right)^{-1}, \\
F^{\prime}=(2 \pi)^{3} \delta(\vec{p}-\vec{q}) E_{p}^{+} .
\end{gathered}
$$

Поскольку уровни энергии ВП-атома определяются полюсами полной функции Грина как функции $E$, а основное взаимодействие между частицами кулоновское, то вблизи полюсов энергия связи $W_{n}=-\mu \alpha^{2} /\left(2 n^{2}\right)$, а функция $F$ выражается через нерелятивистскую функцию Грина свободной частицы $f$ соотношением

$$
F=(2 \pi)^{3} \Omega_{p} \delta(\vec{p}-\vec{q}) f, \quad f=-2 \mu\left(p^{2}+\gamma_{n}^{2}\right)^{-1}, \quad \gamma_{n}^{2}=\frac{\mu^{2} \alpha^{2}}{n^{2}} .
$$

Таким образом, положительно-частотная часть функции $\widehat{G}_{0}$ оказывается полюсной и обеспечивает наибольший вклад в разложение для $\widehat{G}_{c}$. При малых импульсах $\vec{p}^{2} \ll$ 
$m_{i}^{2}$ можно учитывать лишь большие компоненты спиноров. В результате функция $\widehat{G}_{c}^{+}$ может быть разложена в ряд,

$$
\widehat{G}_{c}^{+}=f(\vec{p})\left[\delta\left(\vec{p}_{q}\right)+v_{c}\left(\vec{p}_{q}\right) f(\vec{q})+v_{c}\left(\vec{p}_{k}\right) f(\vec{k}) v_{c}\left(\vec{k}_{q}\right) f(\vec{q})+\cdots\right] .
$$

Члены этого ряда имеют одинаковый порядок по $\alpha$, и вблизи полюсов функция $\widehat{G}_{c}^{+}$ведет себя так же, как функция Грина уравнения Шредингера с кулоновским потенциалом $g_{c}^{-1}=f^{-1}-v_{c}$. Таким образом, учет многократного обмена кулоновскими фотонами необходим только в низкочастотной (НЧ) области, где может эффективно применяться нерелятивистское приближение. Выполнить суммирование полюсных членов в выражении для квазипотенциала можно, используя формулы

$$
\begin{aligned}
& \left(Q G_{c}\right)^{+}=\left(Q \Sigma \Gamma_{0}\right)^{+} \widehat{G}_{c}^{+}, \quad \Sigma=\left(I+\Lambda^{--} F^{\prime} v_{c}\right)^{-1}, \\
& \left(G_{c} Q\right)^{+}=\widehat{G}_{c}^{+}\left(\Sigma^{\prime} \Gamma_{0} Q\right)^{+}, \quad \Sigma^{\prime}=\left(I+v_{c} F^{\prime} \Lambda^{--}\right)^{-1},
\end{aligned}
$$

где $Q$ - произвольный набор операторов.

Рассмотрим блок квазипотенциала

$$
\tilde{\tau}_{c}=\left[\widehat{G}_{c}^{+}\right]^{-1} \widehat{G_{c} \widetilde{T} G_{c}^{+}}\left[\widehat{G}_{c}^{+}\right]^{-1}
$$

С помощью выражений (17) находим

$\left[\widehat{G}_{c}^{+}\right]^{-1}=F^{-1}-K_{c}^{+}-\delta K_{\Sigma}, \quad \delta K_{\Sigma}=-u_{1}^{*} u_{2}^{*} v_{c} \Lambda^{--} F^{\prime} v_{c} u_{1} u_{2}, \quad K_{c}^{+}=u_{1}^{*} u_{2}^{*} v_{c} u_{1} u_{2}$.

В нерелятивистском случае можно учитывать лишь проекции на положительно-частотные состояния и пренебречь билинейными по импульсам членами. Поэтому

$$
\left[\widehat{G}_{c}^{+}\right]^{-1} \approx g_{c}^{-1}, \quad g_{c}=f\left(1+v_{c} g_{c}\right) .
$$

Рассмотрим далее основную часть амплитуды $\tilde{\tau}_{c}$,

$$
G_{c} \widetilde{T} G_{c}=\left[I+G_{0} T_{c}\right] G_{0} \tilde{T} G_{0}\left[I+T_{c} G_{0}\right],
$$

где

$$
\begin{gathered}
G_{0}\left(\vec{k}, \vec{k}^{\prime} ; E\right)=i(2 \pi)^{4} \delta^{4}\left(\vec{k}-\vec{k}^{\prime}\right) S_{1}\left(E_{1}+k_{0}, \vec{k}\right) S_{2}\left(E_{2}-k_{0},-\vec{k}\right), \quad E_{i}=\eta_{i} E, \quad i=1,2, \\
S_{i}\left(p_{0}, \vec{p}\right)=\left[\Lambda_{i}^{+}(\vec{p})\left(p_{0}-\varepsilon_{i p}+i 0\right)^{-1}+\Lambda_{i}^{-}(-\vec{p})\left(p_{0}+\varepsilon_{i p}-i 0\right)^{-1}\right] \gamma_{i 0}, \\
\eta_{1}=\frac{E^{2}+m_{1}^{2}-m_{2}^{2}}{2 E^{2}}, \quad \eta_{2}=\frac{E^{2}+m_{2}^{2}-m_{1}^{2}}{2 E^{2}}
\end{gathered}
$$

Учитывая лишь проекции на положительные частоты, имеем

$$
\begin{aligned}
\widehat{G_{0} \widetilde{T} G_{0}} & =-(2 \pi)^{-4} \Lambda^{++}(\vec{k}) \Gamma_{0} F^{+}(\vec{k}) \widetilde{T}\left(\vec{k}, \vec{k}^{\prime} ; E\right) F^{+}\left(\vec{k}^{\prime}\right) \Lambda^{++}\left(\vec{k}^{\prime}\right) \Gamma_{0}= \\
& =u_{1}(\vec{k}) u_{2}(-\vec{k}) F^{+}(\vec{k})\left[\widetilde{T}\left(\vec{k}, \vec{k}^{\prime} ; E\right)\right]_{+} F^{+}\left(\vec{k}^{\prime}\right) \bar{u}_{1}\left(\vec{k}^{\prime}\right) \bar{u}_{2}\left(-\vec{k}^{\prime}\right),
\end{aligned}
$$

где

$$
\begin{aligned}
{\left[\widetilde{T}\left(\vec{k}, \vec{k}^{\prime} ; E\right)\right]_{+} } & =\bar{u}_{1}(\vec{k}) \bar{u}_{2}(-\vec{k}) \widetilde{T}\left(\vec{k}, \vec{k}^{\prime} ; E\right) u_{1}(\vec{k}) u_{2}(-\vec{k}) \\
\widetilde{T}\left(\vec{k}, \vec{k}^{\prime} ; E\right) & =\left.T\left(k_{0}, k_{0}^{\prime}, \vec{k}, \vec{k}^{\prime} ; E\right)\right|_{k_{0}=k_{0}^{\prime}=0}
\end{aligned}
$$




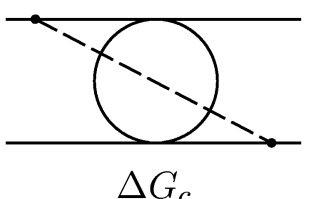

$\Delta G_{c}$

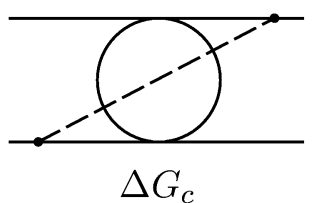

Рис. 1

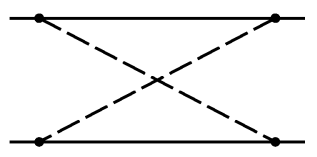

Результатом преобразования (23) является суммирование приводимых диаграмм с кулоновскими линиями в НЧ-области. Целесообразность определения квазипотенциала через $\tilde{\tau}_{c}$ проявляется здесь весьма наглядно.

Итак, в НЧ-области квазипотенциал [13], [14] имеет вид

$$
\begin{aligned}
V(\vec{p}, \vec{q} ; E) & \cong\left[\widehat{G}_{c}^{+}\right]^{-1} \widehat{G_{c} \widetilde{T} G_{c}^{+}}\left[\widehat{G}_{c}^{+}\right]^{-1}-\left[\widehat{G}_{c}^{+}\right]^{-1} \widehat{G_{c} \widetilde{T}} G_{c}^{+}\left[\widehat{G}_{c}^{+}\right]^{-1} \widehat{G_{c} \widetilde{T} G_{c}^{+}}\left[\widehat{G}_{c}^{+}\right]^{-1} \ldots= \\
& =\widetilde{T}_{+}(\vec{p}, \vec{q} ; E)-\widetilde{T}_{+}(\vec{p}, \vec{k} ; E) g_{c} \widetilde{T}+(\vec{k}, \vec{q} ; E) .
\end{aligned}
$$

В низшем приближении $\widetilde{T}=K^{(1)}+K^{(2)}$, ядро $K^{(1)}=K_{T}$ характеризует обмен поперечным фотоном, ядро $K^{(2)}[14]$ изображено на рис. 1.

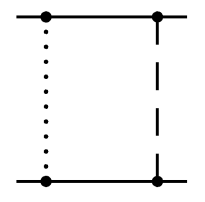

a
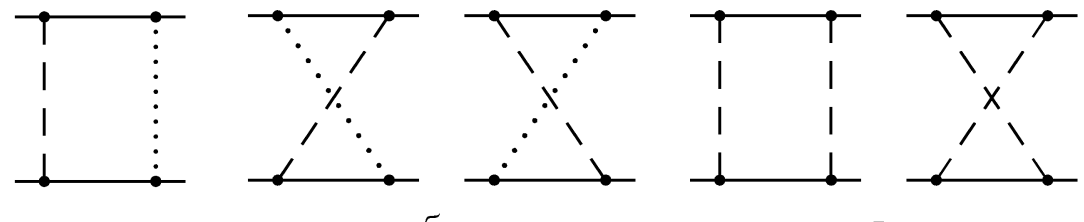

Рис. 2

В высокочастотной (ВЧ) области функция Грина $G_{c}$ разлагается в ряд, и при исследовании двухфотонных обменов квазипотенциалу соответствуют диаграммы, изображенные на рис. 2. Нижняя граница ВЧ-области определяется параметром $\varepsilon$, который выбирается из условия $\mu \alpha^{2} \ll \varepsilon \ll \mu$. Поправка к уровням энергии мюония от приводимых на рис. 2а диаграмм [15] определяется в этой области выражением

$$
\Delta E_{\mathrm{a}}^{\mathrm{Bu}}=\left\langle\varphi_{c}^{\prime}\left|\left[K_{c} G_{0} K_{T}+K_{T} G_{0} K_{c}\right]_{0 F}^{+}\right| \varphi_{c}^{\prime}\right\rangle-\left\langle\varphi_{c}^{\prime}\left|\left[K_{T}\right]_{0 F}^{+}\right| \varphi_{c}\right\rangle-\left\langle\varphi_{c}\left|\left[K_{T}\right]_{0 F}^{+}\right| \varphi_{c}^{\prime}\right\rangle,
$$

где $\varphi_{c}$ и $\varphi_{c}^{\prime}$ определены равенствами (13).

В НЧ-области $0<k<\varepsilon$ все указанные поправки сводятся к расчету однофотонного взаимодействия. Используемая в этом случае нерелятивистская теория приводит к замене радикалов $\varepsilon_{i k}$ массами $m_{i}$ и не дает возможности корректно рассчитать логарифмические по $\beta$ вклады. 
После интегрирования выражения (25) по нулевой компоненте виртуального импульса для вклада в сдвиг $1 S$-уровня от диаграмм двухфотонного обмена находим

$$
\begin{aligned}
\Delta E_{\mathrm{a}}^{\mathrm{Bu}}= & \frac{32 \alpha^{4} \mu^{2}}{(2 \pi)^{5}}\left|\varphi_{c}(0)\right|^{2} \iint d \vec{p} d \vec{q} \varphi_{p}^{2} \varphi_{q}^{2} \Omega_{p} \Omega_{q} \int_{|\vec{k}|>\varepsilon} \frac{d \vec{k} F(k)}{\vec{k}_{p}^{2}\left|\vec{k}_{q}\right|} u_{1}^{*}(\vec{p}) u_{2}^{*}(q) \times \\
& \times\left\{\frac{2 \Lambda^{++}(\vec{k}) E_{k}^{-}}{\left|\vec{k}_{q}\right|}+\Lambda^{--}(\vec{k}) E_{k}^{+}\left[F_{k q}^{1}+F_{k q}^{2}\right]-F_{k q}^{1} \Lambda^{-+}(\vec{k}) \times\right. \\
& \left.\times\left(\varepsilon_{1 q}+\varepsilon_{2 k}+\left|\vec{k}_{q}\right|-E\right)^{-1}-F_{k q}^{2} \Lambda^{+-}(\vec{k})\left(\varepsilon_{2 q}+\varepsilon_{1 k}+\left|\vec{k}_{q}\right|-E\right)^{-1}\right\} \times \\
& \times \Gamma_{0}\left[\vec{\gamma}_{1} \vec{\gamma}_{2}-\left(\vec{k}_{q} \vec{\gamma}_{1}\right)\left(\vec{k}_{q} \vec{\gamma}_{2}\right) \vec{k}_{q}^{-2}\right] u_{1}(\vec{q}) u_{2}(-\vec{q}),
\end{aligned}
$$

где

$$
F_{k q}^{i}=\left(\varepsilon_{i k}+\varepsilon_{i q}+\left|\vec{k}_{q}\right|\right)^{-1}, \quad E_{k}^{-}=\left(\varepsilon_{1 k}+\varepsilon_{2 k}-E\right)^{-1} .
$$

Эта величина не содержит особенностей в НЧ-области, если $\vec{p}, \vec{q} \neq 0$, а $E \neq m_{1}+m_{2}$. Отделим в подьнтегральном выражении (26) члены, приводящие к вкладам, зависящим только от константы тонкой структуры, т.е. те, которые могли быть учтены при получении традиционного результата (10) с помошью уравнения Бете-Солпитера [11], [12] или других методов, включая квазипотенциальный подход [14], [16]. В оставшемся после этого интеграле можно перейти к пределу $\varepsilon \rightarrow 0$. Основной интеграл, необходимый для расчета логарифмических по $\beta$ поправок, принимает в результате вид

$$
\Delta E_{\mathrm{a}}^{\beta}=\frac{8 \alpha^{7} \mu^{3} \beta \mu}{(2 \pi)^{6}(1+\beta)} \int \frac{d \vec{k}}{\varepsilon_{k 1} \varepsilon_{k 2}} \varphi_{k} \frac{M_{k 1} M_{k 2}^{2}}{\left(M_{k 1}+M_{k 2}\right)} J_{p k} J_{q},
$$

где

$$
\begin{gathered}
J_{p k}=\int \frac{d \vec{p}}{\vec{k}_{p}^{2}} N_{p 12} \varphi_{p}^{2}, \quad J_{q}=\int d \vec{q} N_{q 12} \varphi_{q}^{2}=\frac{1}{m_{2}} \int_{0}^{\infty} \frac{d q q^{2}}{\left(q^{2}+\gamma^{2}\right)^{2}} N_{q}(1) N_{q}(\beta), \\
N_{q}(\beta)=\sqrt{\frac{\sqrt{q^{2}+\beta^{2}}+\beta}{2 \sqrt{q^{2}+\beta^{2}}}}, \quad \gamma=\beta \delta, \quad \delta=\frac{\alpha}{(1+\beta)}, \\
N_{p 12}=N_{p 1} N_{p 2}, \quad N_{p i}=\sqrt{\frac{M_{p i}}{2 \varepsilon_{p i}}} .
\end{gathered}
$$

Для вычисления сдвига (27) необходимо вычислить интеграл $J_{p k}$. Сначала рассмотрим более простой интеграл $J_{q}$, который представим в виде

$$
J_{q}=\frac{1}{m_{2}} \int_{0}^{\infty} \frac{d q q^{2}}{\left(q^{2}+\gamma^{2}\right)^{2}}\left[1-\left(1-N_{q}(1)\right)\right] N_{q}(\beta) .
$$

В любом случае $N_{q}(\beta) \leqslant 1$, но при $\beta=0$ имеем $N_{q}(\beta)=1 / \sqrt{2}$, а при $q=0$ будет $N_{q}(\beta)=1$. В то же время основной вклад интеграла

$$
\int_{0}^{\infty} \frac{d q N_{q}(\beta)}{\left(q^{2}+\gamma^{2}\right)}=\frac{\pi}{2 \gamma}
$$


получается при $N_{q}(\beta)=1$, а основной вклад другого интеграла

$$
\int_{0}^{\infty} \frac{d q N_{q}(\beta)}{\left(q^{2}+1\right)}=\frac{\pi}{2 \sqrt{2}}
$$

получается при $N_{q}(\beta)=1 / \sqrt{2}$.

Остановимся на некоторых подробностях вычисления значения $\Delta E_{\mathrm{a}}^{\beta}$. С необходимой для дальнейших расчетов точностью получаем

$J_{q}=\frac{\pi^{2}}{\omega}+\frac{2 \pi}{\mu}[\ln (1+\sqrt{2})-\sqrt{2}]-\frac{\sqrt{2} \pi}{m_{2}(1+\sqrt{2})}[\ln (1+\sqrt{2})-\sqrt{2}]-\frac{\pi \beta}{4 \sqrt{2} m_{2}} \ln \beta^{-1}$.

При расчетах логарифмических вкладов в сдвиг $\Delta E_{\mathrm{a}}^{\beta}$ нас будут интересовать отдельные значения в $J_{q}: J_{1 q}=\pi^{2} / \omega$ и $I_{1 q}=-\pi \beta \ln \beta^{-1} /\left(4 \sqrt{2} m_{2}\right)$. Подставляя $J_{1 q}$ вместо $J_{q}$ в (27), находим

$$
J_{1}=\frac{2 \alpha^{6} \mu^{3}}{(1+\beta)(2 \pi)^{4}} \int \frac{d \vec{k}}{\varepsilon_{k 1} \varepsilon_{k 2}} \varphi_{k} \frac{M_{k 1} M_{k 2}^{2}}{\left(M_{k 1}+M_{k 2}\right)} J_{p k}=\frac{\alpha^{5} \mu^{3}}{2 \pi m_{1} m_{2}} \beta \ln \beta^{-1}\left(\beta \ln \beta^{-1}-1\right) .
$$

Другой вклад в сдвиг (27), содержащий логарифмическую поправку, имеет вид

$$
J_{2}=-\frac{2 \pi \alpha^{7} \mu^{3} \mu \beta^{2}}{\sqrt{2}(2 \pi)^{6} m_{2}} \ln \beta^{-1} \int \frac{d \vec{k}}{\varepsilon_{k 1} \varepsilon_{k 2}} \varphi_{k} \frac{M_{k 1} M_{k 2}^{2}}{\left(M_{k 1}+M_{k 2}\right)} J_{p k}=-\frac{1}{8 \sqrt{2} \pi} \frac{\alpha^{5} \mu^{3}}{m_{1} m_{2}} \beta^{2} \ln \beta^{-1} .
$$

Суммарный логарифмический вклад от диаграмм рис. $2 \mathrm{a}$ дается величиной

$$
\Delta E_{\mathrm{a}}^{\beta}=\frac{\alpha^{5} \mu^{3}}{2 \pi m_{1} m_{2}}\left[-\beta \ln \beta^{-1}+\left(1-\frac{1}{4 \sqrt{2}}\right) \beta^{2} \ln \beta^{-1}\right] .
$$

Отметим, что величина рассматриваемого сдвига может быть рассчитана с подобной точностью лиш при учете нормирующих множителей $N_{p i}, N_{q i}$.

В то же время, в отличие от диаграммы рис. 2б, аналогичный логарифмический по $\beta$ вклад получается и при рассмотрении диаграмм рис. 2в. При этом в ВЧ-области 3-импульсы $\vec{p}, \vec{q}=0$, а $E=m_{1}+m_{2}$, и выражение для амплитуды принимает вид

$$
\begin{aligned}
T_{T T}^{\mathrm{BU}} & =\frac{2 \alpha^{2}}{i \pi^{2}} \int_{|\vec{k}|>\varepsilon} d \vec{k} \int \frac{d k_{0} k_{0}^{2}}{\left(k_{0}^{2}-\vec{k}^{2}\right)^{2}} z_{1}\left(k_{0}\right)\left[z_{2}\left(-k_{0}\right)-z_{2}\left(k_{0}\right)\right]= \\
& =\frac{2 \alpha^{2}}{m_{1} m_{2}}\left[\ln \frac{2 \varepsilon}{\mu}+\frac{m_{1}^{2} \ln \frac{\mu}{m_{2}}-m_{2}^{2} \ln \frac{\mu}{m_{1}}}{m_{1}^{2}-m_{2}^{2}}\right],
\end{aligned}
$$

где $z_{i}\left(k_{0}\right)=\left[\left(m_{i}+k_{0}\right)^{2}-\varepsilon_{i k}^{2}\right]^{-1}$. Инфракрасная особенность устраняется в сумме вкладов от значений амплитуды $T_{T T}$ в НЧ- и ВЧ-областях. При этом в НЧ-области используются лишь большие компоненты биспиноров и полная энергия, как и прежде, полагается равной сумме масс частищ. Отделяя не зависящую от спина часть амплитуды, находим

$$
T_{T T}^{\mathrm{HU}}=-\frac{\alpha^{2}}{2 \pi m_{1} m_{2}} \int_{0}^{\varepsilon} k^{2} d k \int \frac{d \Omega_{k}}{\sqrt{\vec{k}_{p}^{2}} \sqrt{\vec{k}_{q}^{2}}\left[\sqrt{\vec{k}_{p}^{2}}+\sqrt{\vec{k}_{q}^{2}}\right]}\left[1+\frac{\left(\vec{k}_{p} \vec{k}_{q}\right)^{2}}{\vec{k}_{p}^{2} \vec{k}_{q}^{2}}\right] .
$$


Этот интеграл не имеет особенностей при $k=0$, и величина сдвига $\Delta E_{c}^{\mathrm{HЧ}}=$ $\left\langle n S\left|T_{T T}^{\mathrm{H \Psi}}\right| n S\right\rangle$ при изменении $k$ в промежутке $0 \leqslant k \leqslant \omega / n$ имеет порядок $\alpha^{5}$. Если же $k$ меняется в промежутке $\omega / n \leqslant k \leqslant \varepsilon$, можно воспользоваться $\delta$-приближением кулоновских волновых функций,

$$
|n S\rangle=\varphi_{n S}(\vec{p}) \cong(2 \pi)^{3} \varphi_{n S}(0) \delta(\vec{p}) .
$$

Так, при $n=2$ получим

$$
\Delta E_{\mathrm{c}}^{\mathrm{HU}}=-\frac{\alpha^{2}}{2 \pi m_{1} m_{2}}\left|\varphi_{2 S}(0)\right|^{2} \int_{\omega / 2}^{\varepsilon} k^{2} d k \int \frac{d \Omega_{k}}{k^{3}}=\frac{2 \alpha^{2}}{m_{1} m_{2}}\left|\varphi_{2 S}(0)\right|^{2} \ln \frac{\omega}{2 \varepsilon} .
$$

Поскольку согласно (34) величина сдвига $2 S$-уровня в высокочастотной области принимает вид

$$
\Delta E_{\mathrm{a}}^{\mathrm{BU}}=\left\langle n S\left|T_{T T}^{\mathrm{BU}}\right| n S\right\rangle=\frac{2 \alpha^{2}}{m_{1} m_{2}}\left|\varphi_{2 S}(0)\right|^{2}\left[\ln \frac{2 \varepsilon}{\mu}+\frac{m_{1}^{2} \ln \frac{\mu}{m_{2}}-m_{2}^{2} \ln \frac{\mu}{m_{1}}}{m_{1}^{2}-m_{2}^{2}}\right],
$$

то инфракрасные особенности в области $0 \leqslant k \leqslant \infty$ компенсируются и вклад от диаграмм рис. 2в, содержаший $\ln \beta^{-1}$, дается выражением

$$
\Delta E_{\mathrm{c}}=\frac{\alpha^{5} \mu^{3}}{4 \pi m_{1} m_{2}}\left[\beta^{2} \ln \frac{1+\beta}{\beta}-\frac{1}{1-\beta^{2}} \ln (1+\beta)\right] .
$$

Отсюда получаем чисто логарифмические вклады

$$
\Delta E_{\mathrm{c}}^{\beta}=\frac{\alpha^{5} \mu^{3}}{4 \pi m_{1} m_{2}} \beta^{2} \ln \beta^{-1}
$$

для $2 S$-уровня и

$$
\Delta E_{\mathrm{c}}^{\beta}=\frac{2 \alpha^{5} \mu^{3}}{\pi m_{1} m_{2}} \beta^{2} \ln \beta^{-1}
$$

для $1 S$-уровня.

Итак, полученный в квазипотенциальном подходе результат вычисления логарифмического по $\beta$ вклада от диаграмм обмена двумя поперечными фотонами [16] совпадает с аналогичной величиной (10). Это единственная подобная поправка, которая находится в $\delta$-приближении волновых функций (36). Остальные поправки такого рода (например, рассчитанная нами поправка $\Delta E_{a}^{\beta}$ ) в этом приближении обрашаются в ноль и могут быть получены лишь с учетом точных выражений для кулоновских волновых функций и квазипотенциала. Эффективность подобных вычислений демонстрирует изложенный нами трехмерный релятивистский подход к анализу эффектов отдачи в ВП-атомах.

Нами обнаружены и другие поправки, содержащие величины, пропорциональные $\ln \beta^{-1}[17]$. Совокупность этих новых вкладов в сдвиг $2 S$-уровня составляет величину порядка -1.7 Гц для водорода и -8 кГц для мюония. Определенная экспериментально величина сдвига $(1 S-2 S)$-уровней составляет в мюонии [18]

$$
\delta \nu_{1 S-2 S}^{\text {эксп }}=2455528941.0(9.8) \mathrm{MГц,}
$$


а в водороде [6]

$$
\delta \nu_{1 S-2 S}^{\text {эксп }}=2466061413187.34(84) \text { кГц. }
$$

Таким образом, вычисленная нами аналитически разность вкладов для $1 S$ - и $2 S$ уровней не выходит за пределы ошибки эксперимента.

Более сложную картину выявляет сравнение теории и эксперимента применительно к классическому лэмбовскому сдвигу в атоме водорода. Исходя из данных обзора [6], имеем

$$
\begin{aligned}
& \Delta E_{\mathrm{L}}^{\text {теор }}=1057833(4) \text { кГц, } \\
& \Delta E_{\mathrm{L}}^{\text {эксп }}=1057845(3) \text { кГц. }
\end{aligned}
$$

Расхождение теории и эксперимента для лэмбовского с двига составляет не менее 5 кГц. Исправленное с учетом наших расчетов значение величины сдвига $2 S$-уровня энергии в атоме водорода сближает теоретический (39) и экспериментальньй (40) результаты для классического лэмбовского сдвига.

Благодарности. Авторы выражают признательность С. Н. Багаеву, В. И. Саврину и О.А. Хрусталеву за внимание к работе.

\section{Список литературы}

[1] Н. Н. Боголюбов, Д. В. Ширков. Введение в теорию квантованных полей. М.: Наука, 1976.

[2] F. Gross. Phys. Rev. 1969. V. 186. P. 1448.

[3] H. Groth, D. R. Yennie. Rev. Mod. Phys. 1969. V. 41. № 2. P. 350.

[4] A. A. Logunov, A. N. Tavkhelidze. Nuovo Cimento. 1963. V. 29. P. 380.

[5] В. В. Двоеглазов, Ю. Н. Тюхтяев, Р. Н. Фаустов. ЭЧАЯ. 1994. Т. 25. С. 144.

[6] M.I. Eides, H. Grotch, V. A.Sheluto. Phys. Rept. 2001. V. 342. P. 63; hep-ph/0002158.

[7] R.N. Fell. Single transverse photon contribution to the $2 S$ energy level of positronium. Massachusetts: Brandeis University Waltham, 1992.

[8] Ю.Н. Тюхтяев. ТМФ. 1978. Т. 36. С. 264.

[9] Н. Е. Нюнько, Ю. Н. Тюхтяев, Р. Н. Фаустов. ЯФ. 1979. Т. 30. № 2. С. 457.

[10] Н.А. Бойкова, Ю.Н. Тюхтяев, Р. Н. Фаустов. Поправки к сверхтонкому расщеплению основного уровня мюония относительного порядка $m_{e} / m_{\mu} \alpha^{2} \ln \alpha$. В сб.: Труды IV международного семинара по проблемам физики высоких энергий и квантовой теории поля. Т. 1. Ответств. за выпуск С.Н. Соколов. Ред. М.Л. Фоломешкина. Протвино, Серпухов: ИФВЭ, 1983. C. 116.

[11] E.E. Salpeter. Phys. Rev. 1952. V. 87. № 2. P. 328.

[12] T. Fulton, P. C. Martin. Phys. Rev. 1954. V. 95. № 3. P. 811.

[13] Ю. Н. Тюхтяев. ТМФ. 1982. Т. 53. С. 419.

[14] R. N. Faustov. The Dyson equation for the two-particle Green function and the bound state problem. JINR preprint E2-6939. Dubna, 1973.

[15] Ю. Н. Тюхтяев, Р. Н. Фаустов. Поправки к фермиевскому расщеплению основного уровня энергии $\alpha^{2} \ln \alpha$. Сообщ. ОИЯИ Р2-86-281. Дубна, 1986.

[16] Н. Е. Нюнько, Ю.Н. Тюхтяев, Р. Н. Фаустов. Структура протона и тонкое расщепление в атоме водорода. Сообщ. ОИЯИ Р2-7530. Дубна, 1973.

[17] Н. А. Бойкова, Ю. Н. Тюхтяев, Р. Н. Фаустов. ЯФ. 1998. Т. 61. С. 866.

[18] V. Meyer, S. N. Bagaev. Phys. Rev. Lett. 2000. V. 84. P. 1136.

Поступила в редакцию 2.IV.2002 г. 